\title{
Round 4A: Not a Knock-Out Punch, But Rather a Call For Pluralism - A Further Reply to Held
}

\author{
DANIEL B. FISHMAN ${ }^{\mathrm{a}, \mathrm{b}}$ \\ ${ }^{a}$ Graduate School of Applied and Professional Psychology, Rutgers University, Piscataway, NJ \\ ${ }^{\mathrm{b}}$ Correspondence concerning this article should be addressed to Daniel B. Fishman, Graduate School of Applied \\ and Professional Psychology, Rutgers University, 152 Frelinghuysen Road, Piscataway, NJ 08854. \\ Email: $\underline{\text { dfish96198@aol.com }}$
}

\begin{abstract}
This is the fourth round in my interchange with Barbara Held about the nature and role of objectivity and causality as they relate to the rationale for scientific and scholarly legitimacy of case study knowledge. Throughout, I have found Held's critical responses to my views collegially delivered and very helpful in stimulating me to clarify and expand upon my own thinking. In this fourth and final round to our discussion, as context, I include a narrative summary of the back and forth in our discussion during the first three rounds. I then point out that Held's definition of objective knowledge in Round 3 does not separate out a crucial distinction between (a) truth within a knowledge system that is a function of the internal logic of the system, and (b) the attitudes, values, and beliefs of particular individuals and groups. I argue that this distinction plays a very important pragmatic role in our society (e.g., in the functioning of the criminal justice system) in creating knowledge with important characteristics of objectivity - i.e., characteristics associated with the coherence and pragmatic criteria of truth. I also argue that this line of reasoning allows us to distinguish causal mechanisms that are more or less objective in their roles as conceptual tools. I conclude by proposing that broadening the concept of objectivity to include the coherence and pragmatic criteria of truth helps to facilitate acceptance of William James' attractive idea of psychology as a "pluralistic society" rather than as a monolithic knowledge structure.
\end{abstract}

Key words: pragmatism; natural science; positivism; conventional science; objectivity; causality; perspectivism; pluralism

This is the fourth round in my interchange with Barbara Held. Our topic has focused on the nature and role of objectivity and causality as they relate to the rationale for scientific and scholarly legitimacy of case study knowledge in the field of psychology and psychotherapy. I appreciate the collegial atmosphere in which our exchanges have taken place. In this final round to our discussion, I begin by narratively recapitulating our discussion during the first three rounds, and then proceed to my reaction to Held's latest comments. 
Round 4A: Not a Knock-Out Punch, But Rather a Call For Pluralism - A Further Reply to Held

D.B. Fishman

Pragmatic Case Studies in Psychotherapy, http://pcsp.libraries.rutgers.edu

Volume 2, Module 4, Article 6, pp. 1-12, 10-16-06 [copyright by author]

\section{OBJECTIVITY}

\section{Round 1}

In Held's initial, round 1 article (Held, 2006a), she critiqued the epistemology and ontology I had employed in previous writings to provide a rationale for case study knowledge, both from the perspective of epistemology and ontology. In this, she questioned my use of contemporary philosophical pragmatism and social constructionism in contrast to her preferred positions of conventional, "realist" psychological science. Specifically, within the realist science paradigm, Held defines "objective knowledge" to mean knowledge that is "true" independent of the attitudes and interpretation of any particular person or group (what Held after Ronald Miller calls a "language community"). Held (2006a) illustrates by applying this view of objectivity to causal generalities:

Causal generalities/claims (e.g., when you use intervention $\mathrm{X}$ in a type of case $\mathrm{Y}$, in which conditions $\mathrm{C}$ prevail, you are likely — but not guaranteed - to get outcome Z) exist as causal generalities/claims independently of anyone's beliefs about their nature and/or anyone's moral views about the desirability of outcome $\mathrm{Z}$ in the first place. They may therefore be said to exist objectively in just that (somewhat trivial ontological) sense, even though they may be refined or even completely overturned upon further observation, and they may not extend successfully to new cases or contexts despite all due/warranted expectations/prediction.

Of course, without the random assignment of participants in conventional experimental outcome studies (and thus the elimination of confounding variables), it is possible that something other than intervention $\mathrm{X}$ is the actual cause of outcome $\mathrm{Z}$. Nonetheless, the fundamental epistemological point is this: that we must decide in advance (a) what categories to study based on our interests, (b) what methods to use to conduct that study, and (c) what therapeutic outcomes we value, does not in principle or automatically deprive the causal generalities/claims that emerge inductively from careful (i.e., "adjudicated," to stay with the quasi-judicial analogy) clinical observation of an objective truth status (i.e., of objectivist epistemic warrant). That is, the truth of the causal generalities/claims that emerge inductively from the ever-evolving database advocated by Miller and Fishman does not in principle or automatically depend upon anyone's beliefs about the truth of those generalities/claims. If that were so, those generalities/claims could indeed give us only relativistic "truth" rather than the objective truth I believe to be possible in principle [last set of italics added]. (p. 17-18).

\section{Round 2}

In my round 2 response (Fishman, 2006a) to Held, I began by summarizing three types of "truth" differentiated by philosophers:

(a) "correspondence" or "ontological" truth, which defines a statement as true to the extent that it mirrors the external world (Rorty, 1978), that is, it captures the actual nature of the ontological "stuff" of which the world is made; 
Round 4A: Not a Knock-Out Punch, But Rather a Call For Pluralism - A Further Reply to Held

D.B. Fishman

Pragmatic Case Studies in Psychotherapy, http://pcsp.libraries.rutgers.edu

Volume 2, Module 4, Article 6, pp. 1-12, 10-16-06 [copyright by author]

(b) "coherence" truth, which defines a statement as true within a particular "knowledge system" to the extent that it is consistent with other elements in that system. Examples are a Wittgensteinian language game in which one can make an idiomatic statement that is correct usage, even though the meaning of the statement is not deducible from the combined meanings of the individual words that make it up; a mathematical system like geometry in which a geometric proof can be true or false; a defined body of case law in which a present case can be consistent or inconsistent with that body; or a statement about the fictional world created by a novel that can be true or false in terms of consistency with that world); and

(c) "pragmatic" truth, which defines a statement as true to the extent that it is helps us to cope and solve particular problems and achieve particular goals in today's world. In the pragmatist Charles Peirce's framing, pragmatic truth is defined by its effects: "Let us ask what we mean by calling a thing hard. Evidently that it will not be scratched by many other substances. The whole conception of this quality lies in its conceived effects" (Hartshorne, Weiss, \& Burks, 1958, p. 400).

I then pointed out that while pragmatic psychology embraces both coherence truth and pragmatic truth, it rejects the possibility in the real, physical world (although not necessarily in the world of logical possibility) of correspondence truth. The rejection of correspondence truth stems from the social constructionist epistemology that underlies pragmatic psychology, namely, the foundational position that the physical world cannot be viewed independent of a particular perspective. (Note that "the world" to which I am referring here is the actual physical world, not the world of logical possibility.)

A crucial point in my argument was that since statements that are true within both the coherence and pragmatic theories of truth are true independent of any particular community of knowers, by Held's standard I found statements that are "true" within both the coherence and pragmatic models are objective statements. In my words at that point in the argument:

Thus coherence-based truth of Statement S1 in Knowledge System $\mathrm{K}^{1}$ is derived from its logical coherence with other elements in $\mathrm{K}^{1}$ (that is, the inner logic of $\mathrm{K}^{1}$ ), and therefore is not dependent upon the views of any particular community of knowers. Likewise, the pragmatism-based truth of Statement S2 in Knowledge System $\mathrm{K}^{2}$ is derived from its capacity to be helpful in solving particular problems in particular situations, as evidenced by various kinds of empirical evidence of helpfulness (quantitative and/or qualitative, experimental an/or case-based). The pragmatic knowledge system involved contains criteria of what constitutes proper goals for change, evidence of that change, and an appropriate explanation of how S2 creates desired outcomes in terms of that evidence. Thus, the truth of $\mathrm{S} 2$ is a function of the inner logic of these criteria and associated data interpreted with this knowledge system, and thus is not dependent upon the views of any particular community of knowers. (Fishman, 2006a, p. 6).

In sum, in round 2 I argued that since pragmatic psychology recognizes the coherence and/or pragmatic truth of statements within the logic and parameters of a specified knowledge system as a function of the system per se, such statements are objectively true in that they are 
Round 4A: Not a Knock-Out Punch, But Rather a Call For Pluralism - A Further Reply to Held

D.B. Fishman

Pragmatic Case Studies in Psychotherapy, http://pcsp.libraries.rutgers.edu

Volume 2, Module 4, Article 6, pp. 1-12, 10-16-06 [copyright by author]

independent of the attitudes and interpretations any particular individual or group. For example, an individual or community might decide that geometry is not worth teaching in public school because it is not relevant to the lives of students, and/or the community might decide that there are more elegant mathematical systems to teach than geometry. However, this devaluation of geometry by particular individuals or groups does not change the truth of propositions within the knowledge system of geometry. Once individuals or a group enter that system, the laws of truth and falsity apply to all seekers of knowledge within the system, regardless of their personalities or subjective points of view.

\section{Round 3}

In round 3, Held (2006b) responded to my round 2 statement on objective truth and pragmatic psychology by expanding on her definition of objectivist epistemology from round 1. Specifically, her round 3 position on objectivity is summarized in the following quote:

Let me begin by saying that "my" definition of an objectivist epistemology is one that I have indeed adopted, but it is not one that I constructed. Many contemporary philosophers have put forth some version of it, and in addition to the definition I gave - that "the truth of (or warrant for) a claim does not depend on (or is not relative to) anyone's beliefs about the truth of (or warrant for) a claim" (Held, 2006a, p. 28) - they include the notion that objective knowledge does not depend on any particular perspective, paradigm, conceptual scheme, culture, language, theory, discursive community, and so forth (see Erwin, 1997, 1999; Haack, 2002, 2003; Rescher, 1997; Siegel, 2004; Smith, 2004; Thomasson, 2003). For example, according to Erwin (1999): "An "objectivist epistemology" is one which holds that propositions are generally true or false independently of any particular paradigm, or school of thought, or language, or indeed of what any human believes; and furthermore, that they can often be warranted independently of what anyone believes." (Held, 2006b, p. 2)

\section{Round 4}

Held's (2006b) definition above of an objective statement appears to rule out my proposal in round 2 that objective statements are those that are true within a particular "knowledge system," for such a system is fairly synonomous with the "paradigm, conceptual scheme, culture, language, [or] . . . theory" (p. 2) to which Held refers. On the other hand, Held links together such knowledge systems and "discursive communities," and quotes Erwin (1999) as linking together such knowledge systems ("any particular paradigm, or school of thought, or language") and "what anyone believes" (p. 353).

In contrast, in my round 2 statement, truth within a knowledge system was a function of the internal logic of the system and contrasted with the attitudes, values, and beliefs of particular individuals and groups, which I would interpret to include what Held calls "discursive communities" and what Erwin calls "what any human believes." My concept of a knowledge system was explicitly set forth as a way to separate the internal assumptions and logic of a particular paradigm, culture, or language from the attitudes and beliefs of any particular individual or group of individuals. An example of the contrast between a knowledge system and 
Round 4A: Not a Knock-Out Punch, But Rather a Call For Pluralism - A Further Reply to Held

D.B. Fishman

Pragmatic Case Studies in Psychotherapy, http://pcsp.libraries.rutgers.edu

Volume 2, Module 4, Article 6, pp. 1-12, 10-16-06 [copyright by author]

the beliefs of a group of individuals is the contrast between the logic and procedures of the U.S. criminal justice system and the attitudes and beliefs of individual lawyers and judges who function within that system. True, sometimes the line between the justice system per se and the role of particular individuals in the system becomes blurred (as in the politics of choosing Supreme Court Justices). However, more often the justice system - with its administrative and procedural rules, including levels of appeal; its constitutional and legislative laws; and its established precedents - allows "true" decisions to be determined, where "true" is defined as valid with the parameters of the U.S. justice system, independent of the personal beliefs per se of the particular individuals who occupy the roles of prosecution, defense, and judge.

If Held's (and Erwin's) main point is that it is possible to determine that a statement is objectively true independent of any paradigm, language, and culture, then we bump up against the major divide between modern epistemologies for which Held argues, and the postpositivist and postmodern epistemologies for which I argue. For example, as I had cited in my round 2 response, the work of such postpositivistic philosophers as Popper, Kuhn, Quine, Feyerabend, and Wittgenstein emphasizes the basic impossibility of ontologically objective, scientific knowledge. This is

because of our embeddedness in the logical, cultural, cognitive, and linguistic preconditions of ... [the] knowledge that we do have] - preconditions that change according to historical and cultural context. For Popper, these preconditions include the deductive theoretical principles that we simply have to assume without being able to prove them [e.g., the principle of "falsifiability"]; for Kuhn, the preconditions are scientific paradigms; for Quine and Feyerabend, they are webs of belief; and for Wittgenstein, they are [the structures and rules of] language games.

We can never step out of these preconditions and see the world objectively [in the ontological sense]; for our ability to "see" is contingent upon these preconditions being in place. This notion - that the seeking of knowledge is limited by the need to assume preconditions to that knowledge which can't be proven - is very similar to the linguistic concept of the hermeneutic circle. . . According to the hermeneutic circle, to understand a strange culture, practice, theory, language, and so forth, interpretation occurs within a circle in which the parts are always interpreted within some understanding of the whole, which in turn is understood by coming to understand the constituent parts. Thus, to understand something new requires reference to something that is already known and has to be taken for granted in order to understand the new; and then in turn we must assume the former, the new learning, to analyze and understand critically the latter, what we originally knew and assumed (Fishman, 1999, pp. 87-88).

On the other hand, I would contend that the capacity to establish "objective" truth within a knowledge system - when truth is defined in terms of coherence and pragmatics as I described in my response 2 - is itself of tremendous practical importance. (Note that I place the word "objective" in quotes to acknowledge Held's point that technically, most philosophers reserve the term without quotes to statements that are objectively true in terms of correspondence with the physical word.) This links back to the model of "pragmatic relativism" that Rorty (1989) has 
D.B. Fishman

Pragmatic Case Studies in Psychotherapy, http://pcsp.libraries.rutgers.edu

Volume 2, Module 4, Article 6, pp. 1-12, 10-16-06 [copyright by author]

developed, pointing out that the major knowledge systems that positively dominate our public lives today are

not arbitrary, trivial, or insubstantial. For these frameworks arise from and are embedded in historical traditions and contemporary sociocultural structures and institutions. And even though this does not endow such a framework with absolute authority or certainty [derived from knowledge that ontologically mirrors the world], it does provide the framework with significant momentum and weight in determining the present.

For example, in the industrialized countries of Western Europe, the United States, and Canada over the past 125 years, there have been very strong traditions of striving towards democracy and social justice that are a major moral force in the world today - traditions that can be traced at least back in part to situations and events such as Periclean Greece in the fifth-century B.C., the Magna Carta in 1215, and the Declaration of Independence in 1776. On the other hand, these traditions don't carry objective or absolute moral authority outside of the historical and contemporary sociopolitical context of the last 125 years. The counterexamples of Nazi Germany and fascist Italy in the 1930s and "40s certainly illustrate this lack of absolute moral authority. However, the fact that these two regimes have mainly been the exception over the past 125 years reflects the very substantial force of the democratic and social justice tradition. (Fishman, 1999, p. 113)

\section{CAUSALITY}

\section{Round 1}

In round 1, Held proposes that, based on my previous writings on "bottom up" generalization from groups of case studies, it seems that I am only interested in descriptive, action-oriented generalities across cases, and not in theory, causal mechanisms, or associated general principles of human behavior and psychological change. For example, she suggests that I seem to want "a database of cases in which 'guidelines' or 'rules of thumb' will be set forth without theoretical/causal explanation, ... [ [thinking] it best not to attempt to know why or how a certain approach tended not to help (i.e., produced/caused a beneficial effect) in a certain kind of case" (Held, 2006a, p. 11).

\section{Round 2}

In the second round, I explicitly rejected this interpretation of my pragmatic psychology model. I pointed out that in the main conceptual framework that I employ, Peterson's (1991) Disciplined Inquiry model of professional practice, a crucial component of any case study is the theoretical, "guiding conception" that the practitioner brings to the case. I pointed out that such guiding conceptions should have

(a) theoretical and logical coherence and persuasiveness,

(b) predictive validity in anticipating future behaviors and reported experiences, and 
Round 4A: Not a Knock-Out Punch, But Rather a Call For Pluralism - A Further Reply to Held

D.B. Fishman

Pragmatic Case Studies in Psychotherapy, http://pcsp.libraries.rutgers.edu

Volume 2, Module 4, Article 6, pp. 1-12, 10-16-06 [copyright by author]

(c) practical value in providing guidance in the design and implementation of psychological intervention programs in all areas of applied psychology, including psychotherapy.

(Fishman, 2006a, p. 10-11).

I also stressed that within the context of philosophical pragmatism, the only characteristic that such principles lack is ontological objectivity.

That is, in line with the above discussion, within philosophical pragmatism, these causal mechanisms and principles don't purport to mirror the way the world objectively is, independent of any human perception and interpretation of it. However, the pragmatist does view causal mechanisms and principles as important types of pragmatic conceptual tools for managing in and improving the world of human experience and behavior. (Fishman, 2006a, p. 11)

Bringing this perspective to the history of mainstream, natural-science-based, positivistic psychology, in the past I have reframed the value of the focus in this field to build theories identifying the causal mechanisms behind human behavior and experience:

The positivists have staked out psychometrically sophisticated and innovative methodologies that set high standards for rigorous, critical, and ingenious thinking about the complexities of measuring psychological phenomena. In addition, positivists have developed a rich supply of psychological theories and ideas that explore a variety of the vast array of possible perspectives that can be taken upon human behavior and action. (Fishman, 1999, p. 8)

As an example outside of psychology of this notion of theoretical concepts as conceptual tools, I cited Rorty's example of ontological nature of Newton's universal law of gravitation and three laws of motion, built on the early ideas of Copernicus and Galileo:

Galileo and his followers discovered, and subsequent centuries have amply confirmed, that you get much better predictions by thinking of things as masses of particles blindly bumping against each other than by thinking of them as Aristotle thought of them - animistically, teleologically, and anthropomorphically. They also discovered that you get a better handle on the universe by thinking of it as infinite and cold and comfortless than by thinking of it as finite, homey, planned, and relevant to human concerns . . . These [types of discoveries] are the basis of modern technological civilization. But they do not ... tell us anything about ... the language which nature itself uses, ... [about] the Book of Nature. (Rorty, 1982, p. 116).

In discussing causality, in the second round I also considered a central issue that arose in Held's discussion of Ronald Miller's views on the rationale for case studies - namely, the distinction between free will versus determinism, e.g., looking at a problematic human behavior from the point of view of the causal mechanisms determining it versus the questionable moral choices motivating it. In addressing this distinction I proposed that this distinction can also be viewed in terms of the frame of different conceptual tools for evaluating particular human behaviors: 
D.B. Fishman

Pragmatic Case Studies in Psychotherapy, http://pcsp.libraries.rutgers.edu

Volume 2, Module 4, Article 6, pp. 1-12, 10-16-06 [copyright by author]

The pragmatist does not see a direct contradiction between ... [a] view of cause, which places free will at its center, and the mechanistic view of it that comes from mainstream psychology's adoption of the natural science approach to human behavior, which places determinism at its center. Rather, because the pragmatist does not proffer objective ontological status to either the natural science or humanistic/judicial view of cause, the pragmatist sees these simply as two different sets of perspectives for viewing a particular set of behavioral and experiential events. When to use each is a question of the goals and values of the users and how these concepts facilitate or retard those goals and values. (Fishman, 2006a, p. 12)

\section{Round 3}

In round 3 Held is "pleased to know that Fishman agrees that there are what he calls 'causal mechanisms,', but she still views as anti-objectivist my concept of causal mechanisms as conceptual tools. She states that

Since my definition of epistemological objectivity goes hand in hand with (i.e., depends upon) ontological objectivity, that is another reason why Fishman's epistemology is not objectivist in my sense. What can it mean to say that we have objective, or beliefindependent, knowledge of an entity that does not exist independently of our beliefs about its existence and nature? For example, if there is no way that therapy works independently of our beliefs about how therapy works, then we cannot know the workings of therapy objectively. (See Held, 2002, 2006b, and Thomasson, 2003, for arguments about how there can be objective knowledge of mind-dependent entities, such as social/psychological kinds.) (Held, 2006b, p. 4)

She extends this critique to my view of free will versus determinism as different conceptual tools for grappling with the world, and not a reflection of how the world "really" is, that is, in my words, "it all depends on how you look at it." In her words,

Similarly, he [Fishman] makes free will and determinism-traditional causal matters, to be sure - dependent on "how you look at it" (p. 19); for him there seemingly is no way that these causal entities are, independent of beliefs about how they are. If "how you look at it" depends on your (or any other knower's) particular knowledge system, with all the beliefs about causality that may be contained therein, then there can be no objective knowledge of free will and determinism by my definition. (Held, 2006b, p. 5)

\section{Round 4}

Following the tripartite view of truth - correspondence, coherence, and pragmatic - that I summarized in round 2, Held makes it clear that she reserves the term "objective knowledge" to statements that correspond to the external world. She does not -- as I do -- include in her definition of objective knowledge statements that have logical coherence and pragmatic usefulness in coping with the external world within particular knowledge systems (although not documented correspondence with the external world). By her definition, out of many possible, contrasting causal mechanisms to explain a psychological phenomenon, we seek the one that corresponds to the objective, ontological reality of the phenomenon. A statement about this 
Round 4A: Not a Knock-Out Punch, But Rather a Call For Pluralism - A Further Reply to Held

D.B. Fishman

Pragmatic Case Studies in Psychotherapy, http://pcsp.libraries.rutgers.edu

Volume 2, Module 4, Article 6, pp. 1-12, 10-16-06 [copyright by author]

mechanism would be objectively true, and statements about the other causal mechanisms would be objectively false. Likewise, she sees my position that free will and determinism are different conceptual tools, to be judged by their internal coherence and ability to solve difficulties in particular, problematic situations, as "anti-objectivist," ruling out any "objective knowledge of free will and determinism by my [Held's] definition.” (Held, 2006b, p. 5)

\section{CONCLUSION}

Two major, related themes run throughout the four rounds of discussion in which Barbara Held and I have engaged about the nature of objectivity and causality - particularly as they relate to the rationale for the legitimacy of case-study-based knowledge. First, Held argues that a statement can only be objectively true if it corresponds to the nature of the real world. Within my social constructionist epistemology, I argue that such correspondence-based knowledge is not possible, since within this epistemology, we can only know the world through a particular perspective/pair of glasses, and we can never take off the glasses and see the world "as it really is." However, I argue that by other definitions of objective truth - i.e., truth as defined by logical coherence and pragmatic usefulness within a particular knowledge system - statements can be objectively true as judged within that knowledge system.

Second, in looking at knowledge situations that don't meet the correspondence criterion of objective truth, Held does not make a distinction between (a) statements that are logically and/or pragmatically true within a particular knowledge system, independent of any particular individual's or group's beliefs and attitudes, and (b) statements that are held to be true based on the personal beliefs and attitudes of particular individuals or groups. In round 2, I argued that, as summarized in Rorty's (1989) concept of "pragmatic relativism," this distinction is of enormous importance pragmatically, since the major knowledge systems that positively dominate our public lives today, for example the criminal justice system in the United States, are not

arbitrary, trivial, or insubstantial [as many personal beliefs are]. For these frameworks arise from and are embedded in historical traditions and contemporary sociocultural structures and institutions. And even though this does not endow such a framework with absolute authority or certainty [as would knowledge that is proven to ontologically mirror the world], it does provide the framework with significant momentum and weight in determining the present. (Fishman, 1999, p. 113; as quoted in Fishman, 2006a, p. 9)

On the other hand, as I mentioned at the end of the round 2, while conventional science and pragmatism disagree on the possibility of ontologically true knowledge, in many ways this disagreement does not affect the usual conduct of psychotherapy research and practice. For both conventional science and pragmatism endorse: "(a) the development of more and more effective therapies; (b) the documentation of effective therapies with quantitative and qualitative, process and outcome evidence; and (c) the development of more and more, logically and empirically persuasive causal principles of therapeutic change" (Fishman, 2006a, p. 13). Moreover, while pragmatists don't endorse the search for correspondence-based truth as conventional, positivistically oriented scientists do, pragmatists are as concerned about developing and implementing methods to ensure the quality of knowledge that they accumulate, independent of 
Round 4A: Not a Knock-Out Punch, But Rather a Call For Pluralism - A Further Reply to Held

D.B. Fishman

Pragmatic Case Studies in Psychotherapy, http://pcsp.libraries.rutgers.edu

Volume 2, Module 4, Article 6, pp. 1-12, 10-16-06 [copyright by author]

the content of the knowledge. For example, some of the case study methodological controls that I have advocated and that I urge case study authors in this journal to employ include:

(a) a write-up that takes place within a standardized, explicit analytic framework which logically separates descriptive clinical material from theory, which shows the logical links between clinical material and theory, and which facilitates comparisons among cases; (b) a write-up that contains extensive, illustrative quotes from therapy transcripts which provide the basic data upon which inferences are based; (c) a write-up that contains third party observers and interpreters for reliability; and (d) a write-up that contains the use of standardized, quantitative questionnaires before, during, and after therapy so as to place the individual case in a normative framework for comparison with similar types of clients.

(Fishman, 2006b, pp. 5-6)

Still, as I suggested at the end of round 2, the narrower definition of objective truth within conventional science does seem to limit conventional science's lack of openness to "research methodologies that are outside the boundaries of experimental, quantitative group designs [e.g., qualitative and case-based designs] because conventional science views knowledge generated by these experimental designs as ontologically privileged" (Fishman, 2006a, p. 13). In fact, consider the assumptions of the conventional psychological science model - that external reality ontologically consists of a world of discrete, operationalizable variables that are woven together into universal causal laws that can be framed in quantitative terms. I would argue that these assumptions go against many of the qualities of our everyday, personal experience - e.g., qualitative consciousness, moral decision-making, and narrative meaning patterns. This is not to conclude that the conventional psychological science model is an ontologically wrong picture of psychological phenomena. It is simply to assert that the conventional psychological science model seems not to clearly capture the way the "real" world of psychological phenomena is. Experienced. Thus, the contrast between a world of networks of discrete, quantified variables embedded in abstract theories and a world of everyday, qualitatively rich conscious experience would seem to parallel Rorty's example of the ontological status of the physical world assumed by Newton versus Aristotle.

[Newton] discovered, and subsequent centuries have amply confirmed, that you get much better predictions by thinking of things as masses of particles blindly bumping against each other than by thinking of them as Aristotle thought of them - animistically, teleologically, and anthropomorphically. They also discovered that you get a better handle on the universe by thinking of it as infinite and cold and comfortless than by thinking of it as finite, homey, planned, and relevant to human concerns . . . These [types of discoveries] are the basis of modern technological civilization. But they do not ... tell us anything about ... the language which nature itself uses, ... [about] the Book of Nature. (Rorty, 1982, p. 116).

In other words, the advantage of the Newtonian view of the physical world over the Aristotelian view is in the former's capacity to better facilitate the development of modern technology, not in its ontological correspondence to the physical world. Likewise, in terms of the concept of pragmatic relativism, judgments about the "truth" of a particular psychological theory in understanding and/or problem-solving within the realm of certain particular psychological 
Round 4A: Not a Knock-Out Punch, But Rather a Call For Pluralism - A Further Reply to Held

D.B. Fishman

Pragmatic Case Studies in Psychotherapy, http://pcsp.libraries.rutgers.edu

Volume 2, Module 4, Article 6, pp. 1-12, 10-16-06 [copyright by author]

phenomena should be made on the basis of the capacity of the theory to generate accurate empirical predictions and/or effective problem-solving in addressing those phenomena.

Moreover, as I mentioned at the end of round 2, there is an important practical advantage of "objective" knowledge based on coherence and pragmatic truth criteria as opposed to Held's view of objective knowledge based on the correspondence truth criterion. This is the advantage of the former in facilitating multiple approaches in the search for more effective knowledge in addressing the psychological, social, and cultural difficulties that individuals and groups of human beings face. The nature of this approach is reflected in Peterson's (2004) view of psychology as "a pluralistic society" in the context of William James' original vision of the philosophical pragmatism:

To James (1909/1977), the universe was not one but many, everywhere and always. . . Only in the relationships among the parts could unity of the whole be reached. He rejected monistic views as forced distortions of nature. ... The pluralistic world is more like a federal republic than like an empire or kingdom. (Peterson, 2004, p. 2005)

Actually, in closing on the topic of pluralism, I again want to thank Barbara Held for her openness to taking alternative views seriously and for the simultaneously respectful and incisive arguments she has offered in challenging my views. She has been a very helpful motivator to further explore and clarify the nature of my positions.

\section{REFERENCES}

Erwin, E. (1997). Philosophy and psychotherapy: Razing the troubles of the brain. London: Sage.

Erwin, E. (1999). Constructivist epistemologies and therapies. British Journal of Guidance and Counselling, 27, 352-356.

Fishman, D. B. (1999). The case for pragmatic psychology. New York: New York University Press.

Fishman, D. B. (2006a). Round 2A: Finding objectivity and causality in pragmatism -- A response to Held. Pragmatic Case Studies in Psychotherapy [Online], Vol. 2(4), Article 3. Available: http://hdl.rutgers.edu/1782.1/pcsp_journal.

Fishman, D.B. (2006b). From casual "clinical reports" to systematic, peer-reviewed "case studies." The Clinical Psychologist, 59, 5-6.

Haack, S. (2002). Realisms and their rivals: Recovering our innocence. Facta Philosophica, 4, 67-88.

Haack, S. (2003). Defending science-within reason: Between scientism and cynicism. Amherst, NY: Prometheus Books.

Hartshorne, C., Weiss, P., \& Burks, A.W. (Eds.). (1958). Collected papers of Charles Sanders Peirce, vl. 7. Cambridge, MA: Harvard University Press.

Held, B.S. (2006a). Round 1: Does case study knowledge need a new epistemology? This module. 
Held, B.S. (2006b). Round 3: Regarding objectivity and causality -- A rejoinder to Fishman and Miller. This module.

James, W. (1977). A pluralistic universe: Hibbert lectures at Manchester College on the present situation in philosophy. Cambridge, MA: Harvard University Press. (Original work published 1909).

Peterson, D.R. (2004) Science, scientism, and professional responsibility. Clinical Psychology: Science and Practice, 11, 196-210.

Rorty, R. (1978). Philosophy and the mirror of nature. Princeton, NJ: Princeton University Press.

Rorty, R. (1982). Consequences of pragmatism. Minneapolis: University of Minnesota Press.

Rorty, R. (1989). Solidarity or objectivity. In M. Krausz (Ed.), Relativism: Interpretation and confrontation. Notre Dame, IN: University of Notre Dame Press. Also in Rorty, R. (1991). Objectivity, relativism, and truth, pp. 21-34. New York: Cambridge University Press.

Rescher, N. (1997). Objectivity: The obligations of impersonal reason. Notre Dame: University of Notre Dame Press.

Siegel, H. (2004). Relativism. In I. Niiniluoto, M.Sintonen, \& J. Wolenski (Eds.), Handbook of epistemology (pp. 747-780). Netherlands: Kluwer Academic Publishers.

Smith, T. (2004). "Social" objectivity and objectivity of value. In P. Machamer \& G. Wolters (Eds.), Science, values, and objectivity (pp. 143-171). Pittsburgh: University of Pittsburgh Press.

Thomasson, A. L. (2003). Realism and human kinds. Philosophy and Phenomenological Research, 67, 580-609. 